\title{
Knowledge, attitude and practice related to infant feeding among women in rural Papua New Guinea: a descriptive, mixed method study
}

Jerzy Kuzma

\begin{abstract}
Background: Despite the well-recognized effectiveness of exclusive breastfeeding for the first six months of an infant life for reducing infant mortality, adherence to this practice is not widespread in the developing world. Although several studies on infant nutrition practices have been conducted in urban settings of Papua New Guinea (PNG), there is only scant information on infant feeding practices in rural settings. Therefore, this study aimed to investigate knowledge, attitude and practice associated with exclusive breastfeeding in various locations in rural PNG.

Methods: A mixed method study using interviews based on a semi-structured questionnaire $(n=140)$ and Focus Group Discussions (FGDs) was conducted among mothers in rural PNG between August and September 2012. Participants were selected using convenience sampling. Included in the study were both primiparous and multiparous mothers with a child below the age of two years. Content analysis was used for qualitative data and descriptive statistics were used for quantitative data.

Results: Whereas most women indicated breastfeeding as a better way to feed babies, knowledge of the reasons for its superiority over infant formula was generally poor. Only 17\% of mothers practiced exclusive breastfeeding for the first six months postpartum. Our study showed that the size of the gap between exclusive breastfeeding practice and global recommendations was striking. Taking into account the low educational profile of the participants, the disparity may be explained by the fact that most of the mothers in this study had no formal education on infant feeding.

Conclusions: This study showed a lack of understanding of the importance of and poor adherence to exclusive breastfeeding for the first six months postpartum among rural mothers. As exclusive breastfeeding promotion has been proved to be one of most effective ways to improve infant survival, more attention should be given to it, especially targeting the large proportion of women who missed formal education on infant feeding in school. A proper community-based program including the tools for monitoring its implementation and effectiveness needs to be developed to transform policy recommendations into action in rural PNG.
\end{abstract}

Keywords: Exclusive breastfeeding, Bottle feeding, Infant formula, Infant feeding, Papua New Guinea

\section{Background}

Promotion of exclusive breastfeeding practices for the first six months of an infant's life is one of the most effective interventions for reducing infant morbidity and mortality in resource-constrained settings [1]. The World Health Organization (WHO) recommends exclusive breastfeeding for the first six months of life [2,3]. Numerous studies

Correspondence: jkuzma@dwu.ac.pg

Faculty of Health Sciences, Divine Word University, PO Box 483, Madang, Papua New Guinea have underlined the advantages of exclusive breastfeeding for growth, immunity and prevention of illness in young infants [4-6]. Conversely, several studies associate lack of exclusive breastfeeding feeding with high infant mortality and morbidity from malnutrition and infections $[7,8]$.

Despite the well-recognized importance of exclusive breastfeeding, this practice is not widespread in the developing world and the increase at the global level is very modest with much room for improvement $[9,10]$. Infant nutrition programs worldwide continue to require

\section{Biomed Central}

(C) 2013 Kuzma; licensee BioMed Central Ltd. This is an open access article distributed under the terms of the Creative Commons Attribution License (http://creativecommons.org/licenses/by/2.0), which permits unrestricted use, distribution, and reproduction in any medium, provided the original work is properly cited. 
investment and commitment to improve feeding practices in order to have maximum impact on reducing infant morbidity and mortality [10].

Papua New Guinea (PNG) was the first country in the world to introduce legislation to protect breastfeeding with the Baby Food Supplies (Control) Act 1977. This legislation had an immediate impact on the prevalence of infant formula feeding, reducing it in Port Moresby by a third between 1976 and 1979 [10]. However, the impact seemed to be short-lived and a large survey on infant feeding practices in 1995 reported that 20\% of mothers in different parts of PNG had used regularly infant formula [11]. Several studies on infant nutrition practices have been conducted in PNG, predominantly in urban settings [11-13]. However, there is little information on infant feeding practices in the rural setting.

This study aims to investigate the knowledge, attitude, practice and social support connected with infant feeding in various locations of rural PNG.

\section{Methods}

This mixed methods study, with convergent parallel design, was conducted between August and September 2012. To explore the issue deeper and inform the interview questionnaire construction, two Focus Group Discussions (FGDs) were undertaken (with 4 and 8 participants respectively) with women not participating in the interviews. The main topics for the FGDs were practice and customs related to breastfeeding.

Interviews in the PNG language Tok Pisin based on a semi-structured questionnaire which included both openended and closed questions were used to collect information from mothers $(n=140)$ in rural locations of the Eastern Highlands and Madang Provinces. Field workers (university students) were trained by the author to conduct the interviews. Convenience sampling was used to select mothers visiting health centres who had a child of less than two years old. Mothers who were currently or previously working as health workers or who were unable to speak Tok Pisin were excluded. Mothers with infants presenting specific feeding problems (cleft palate or lip, severely ill) were also excluded.

The FGDs and all open-ended questions in the interviews were recorded with a voice recorder and transcribed for analysis, whereas closed-ended questions were manually recorded in the questionnaire. The qualitative and quantitative strands of data were analyzed separately and triangulation was performed at the level of inferences. Content analysis was performed for the qualitative data, and main themes were identified according to common threads in the data. The quantitative data were summarized by descriptive statistics using the mean for continuous and proportions for categorical variables.
The study received ethical clearance from the Divine Word University Research Ethics Committee (No1/3/12).

\section{Results}

\section{Demographic characteristics}

In total 140 mothers with an average age of 29 years (range 18-36 years) participated in the questionnaire study. Demographic characteristics of the sample are shown in Table 1.

\section{Breastfeeding practice}

Ninety eight percent of the mothers $(n=138)$ were breastfeeding their babies but only two-thirds had given colostrum to their babies $(n=96)$. Most explanations given by mothers for refusing to feed their babies colostrum were connected with cultural beliefs that "colostrum may harm the baby". Colostrum was described as "dirty", "unclean", "contained infected pus", "waste from mother's body", "not food for the baby", and "infectious to a child and can cause yellow eyes".

Ninety one percent $(n=128)$ of mothers breastfed their babies when they cried, which amounted to 7-8 times per day. Eighty three percent $(n=116)$ of interviewed mothers supported their babies under six months with additional food. Interestingly, supplementary feeding of babies under six months was more common (88\%) among older ( $\geq$ 30 years of age) than younger mothers (80\%), however this difference was not significant ( $\mathrm{p}=0.23$; Pearson Chi-square test). In the FGDs all mothers admitted starting supplementary food for their babies at three to four months of

Table 1 Demographic characteristics of the study sample

\begin{tabular}{ll}
\hline Characteristic & $\mathbf{n}(\%)$ \\
\hline Ethnicity & $100(71)$ \\
Madang Coastal Province & $40(29)$ \\
Eastern Highlands Province (Okapa District) & \\
Parity & $76(54)$ \\
Primipara & $64(46)$ \\
Multipara & \\
Maternal age & $85(61)$ \\
$<30$ years & $55(39)$ \\
$\geq 30$ years & \\
Maternal education level & $42(30)$ \\
$\leq$ Grade 4 & $62(44)$ \\
Grade 5-8 & $26(26)$ \\
$>$ Grade 8 & $133(95)$ \\
Maternal employment & $7(5)$ \\
Unemployed* & \\
Employed &
\end{tabular}

${ }^{*}$ mothers have no formal job, they are mostly self-employed subsistence farmers. 
age. These included semi-solid food, such as mashed sweet potatoes, papaya, potatoes, banana, pumpkin and soup. Common explanations for early supplementary feeding of their babies were that supplementary food "helps the child to grow well" or that "breast milk is not sufficient for the child to grow and be strong".

More than a half of the mothers $(58 \%, \mathrm{n}=81)$ stopped breastfeeding within 12 months. Of note is that a greater proportion of older mothers $(78 \% ; 40 / 55)$ compared to younger mothers $(35 \%$; 41/85) were still breastfeeding when their baby was 12 months of age $(p=0.06$; Pearson Chi-square test). Factors that influenced the breastfeeding period were: new pregnancy, work demands (gardening, marketing, housework, and professional careers), child choice, needing to go back to their studies, and family problems. Almost two-thirds of mothers $(66 \%, n=92)$ experienced good family support during their breastfeeding period. Qualitative data from the FGDs and open-ended questions in the interview confirmed the quantitative findings.

\section{Attitude towards breastfeeding}

The majority of mothers $(87.9 \%, \mathrm{n}=123)$ regarded breastfeeding as good, giving various reasons for their perception relating to this (Table 2 ).

Less common explanations given in support of breastfeeding were: "it is in line with the culture", "mother shows responsibility for a baby", "breastfeeding is easy no need to prepare food for a baby", and some believed that it prevents pregnancy.

According to mothers, most husbands (99\%, $\mathrm{n}=138$ ) were happy and supportive towards breastfeeding, however $27 \%(n=39)$ said that their husbands had influenced them to shorten the period of breastfeeding. A frequently given explanation for why husbands would have influenced mothers to shorten breastfeeding was a custom that did not allow having sex with a breastfeeding woman. This is a good illustration where the qualitative strand of data provided explanations for the quantitative data.

Table 2 Attitudes of lactating mothers towards breastfeeding

\begin{tabular}{lcc}
\hline Reason* $^{*}$ & $\%$ & $\mathbf{n}$ \\
\hline Healthy for the child & 48 & 67 \\
Helps the child to grow well & 38 & 53 \\
Breast milk is the best food for the child & 36 & 51 \\
Beneficial to the mother (reduces breast tightness and pain) & 63 & 88 \\
Bad for the mother (causes weight loss) & 17 & 22 \\
No known benefit & 23 & 32 \\
\hline
\end{tabular}

*many gave more than one reason.

\section{Food taboos/restriction}

More than half the lactating mothers $(57 \% ; n=80)$ admitted that food restrictions were imposed on them by their culture. In particular a higher proportion of older (80\%) than younger mothers (42\%) admitted food restrictions during lactation. Those restrictions vary in different areas and include fish from the sea, pork fat, pork meat, possum (cuscus), eels, red pandamus fruit (Pandamus conoideus), sugar cane, short banana and taro. Breaking those taboos was believed to cause the child to become sick or crippled, stunted in growth or attain undesirable features of the eaten animal. For instance a mother from the Madang Coast believed that, "if a lactating woman eats bandicoot, her baby would develop tremor and become sick". Another breastfeeding mother stated, "I am not allowed to eat octopus because my baby may develop shortness of breath."

\section{Infant formula feeding practice}

Even though the large proportion of mothers $(77 \%, \mathrm{n}=$ 108) considered infant formula feeding as not good, knowledge about its disadvantages was very low. One third of participants $(30 \%, n=42)$ did not know why infant formula feeding was not good. Interestingly, all mothers lacking knowledge of the hazards associated with infant formula feeding were below Grade 8 educational level. Although $70 \%$ of mothers $(n=98)$ considered that infant formula feeding was not healthy and some noted that it was expensive, very few thought it could cause diarrhoea or illness.

\section{Discussion}

\section{Practice of infant feeding}

A global evidence-based public health resolution recommends exclusive breastfeeding for the first six months of life and continued breastfeeding up to two years of age and beyond [14]. In low-resource countries the prevalence of exclusive breastfeeding at six months is generally low and varies from 9\% [15] to 39\% [16].

Although this study showed that almost all rural mothers (98\%) breastfed their babies, exclusive breastfeeding up to six months of baby's life was not commonly understood and was practiced by only $17 \%$ of mothers. This is consistent with other low-resource countries. The prevalence of breastfeeding in our study differed considerably from findings of a recent study in PNG where $40 \%$ of mothers in urban setting had an experience with infant formula feeding at some stage of child's life [13]. Differences in the employment status of mothers in rural $(5 \%$ employed) and urban (30\% employed) might partially account for the difference.

In our study the prevalence of continued breastfeeding for infants and children aged 12-23 months was average for a low-resource country [2]. The sizable gap 
between breastfeeding practice in developing countries and international recommendations indicates that more attention should be given to the promotion of exclusive breastfeeding. Breastfeeding promotion interventions in developing countries have been shown to result in a sixfold increase in exclusive breastfeeding rates at six months [17]. Furthermore, a recent review of child survival interventions in low-income countries showed that the promotion of breastfeeding was one of the most effective public health interventions, reducing under-five mortality by $13 \%$ [1]. Breastfeeding promotion, therefore, has become a global health priority with maximum impact on both maternal and child health in developing countries.

The HIV epidemic has added another aspect to the importance of best infant feeding practice. For HIV-positive mothers, exclusive breastfeeding for the first six months of a baby's life offers the lowest risk of transmission and child mortality, whereas suboptimal (mixed) feeding is associated with a higher transmission and child mortality rate [18].

\section{Knowledge and attitude towards breastfeeding}

Even though most rural mothers regarded breastfeeding as the best for their babies, knowledge about the benefits of breastfeeding and hazards of infant formula feeding was very low. Taking into account the low educational profile of the participants and the fact that in the current curriculum infant feeding is supposedly to be taught in Grade 8 , this may be explained by the fact that many of the mothers in this study may have missed that formal education on infant feeding.

According to the participants, almost all fathers (99\%) supported breastfeeding; however a quarter of fathers influenced the earlier stopping of breastfeeding. This may be explained by a common belief in PNG that a couple should not resume sexual relations whilst a woman is breastfeeding. A Vietnamese study also found that the husband and maternal mother influenced breastfeeding practice [19]. The majority of the mothers in this study experienced family support for breastfeeding. Another study indicated that peer support increased the duration of exclusive breastfeeding in low and middle-income countries [20].

In this study traditional and customary beliefs influenced infant feeding practice in two areas: feeding with colostrum and imposing various dietary restrictions on lactating women. This finding is consistent with a study of breastfeeding practices in India [21]. Food taboos are known in various cultures and are designed to protect humans from real or assumed health hazards [22]. A better understanding of customary beliefs towards lactating women will allow more effective breastfeeding education and promotion.

\section{Limitations of the study}

There were several limitations in our study. Nonprobability sampling with likely sampling bias, a relatively small sample size and rural locations in only two provinces together with the rich cultural diversity in rural PNG mean that our results cannot be fully extrapolated to the whole rural population.

\section{Conclusions and recommendations}

Rural mothers' knowledge about the benefits of breastfeeding and in particular about the importance of exclusive breastfeeding in a child's first six months of life is poor. This study showed that the size of the gap between exclusive breastfeeding practice in PNG and WHO recommendations is striking.

As exclusive breastfeeding promotions improve infant survival, more attention in health planning should be given to its promotion. In particular, breastfeeding promotion should target the large proportion of women who have missed formal education about infant feeding in school. Greater emphasis should be made at children's and antenatal clinics to educate mothers about the importance of exclusive breastfeeding for the first six months of their infants' life, the advantages of breastfeeding, and potential hazards of feeding a baby with infant formula. Although the PNG National Health Plan 2011-2020 states that to improve child survival, health workers must place more emphasis on exclusive breastfeeding [23], proper planning, including community-based programs and the tools for monitoring its implementation and effectiveness are yet to be developed.

\section{Consent}

Written informed consent was obtained from all participants of the study for the publication of this report.

\section{Competing interests}

The author declares that they have no competing interests.

\section{Acknowledgements}

The author would like to thank the following Divine Word University students for their contribution to data acquisition: Tatireta P, Kavanamur V, Pison J, Mauludu S and Zuvani R. The author wishes also to acknowledge Divine Word University for providing logistic support for the fieldwork.

Received: 12 August 2013 Accepted: 17 November 2013 Published: 21 November 2013

\section{References}

1. Jones G, Steketee RW, Black RE, Bhutta ZA, Morris SS: How many child deaths can we prevent this year? Lancet 2003, 362:65-71.

2. World Health Organization: The Optimal Duration of Exclusive Breastfeeding: Report of an Expert Consultation. Geneva, Switzerland 28-30 March 2001. Geneva; 2002.

3. World Health Organization: Global Strategy for Infant and Young Child Feeding. Geneva: World Health Organization; 2003.

4. Arifeen S, Black RE, Antelman G, Baqui A, Caulfield L, Becker S: Exclusive breastfeeding reduces acute respiratory infection and diarrhea deaths among infants in Dhaka slums. Pediatrics 2001, 108:E67. 
5. Oddy WH, Sly PD, de Klerk NH, Landau L, Kendall G, Holt P, Stanley F: Breast feeding and respiratory morbidity in infancy: a birth cohort study. Arch Dis Child 2003, 88(3):224-228.

6. Kalanda BF, Verhoeff FH, Brabin BJ: Breast and complementary feeding practices in relation to morbidity and growth in Malawian infants. Eur J Clin Nutr 2006, 60(3):401-407.

7. Brown KH, Black RE, Lopez De Romaña G, Creed De Kanashiro H: Infantfeeding practices and their relationship with diarrheal and other diseases in Huascar (Lima), Peru. Pediatrics 1989, 83:31-40.

8. Almeida RM, De Marins VM, Valle J: Breastfeeding, socio-economic conditions and nutritional status of children younger than 12 months in Brazil. Ann Trop Paediatr 1999, 19:257-262.

9. Agampodi SB, Agampodi TC, Piyaseeli UKD: Breastfeeding practices in a public health field practice area in Sri Lanka: a survival analysis. Int Breastfeed J 2007, 2:13.

10. Cai $X$, Wardlaw T, Brown DW: Global trends in exclusive breastfeeding. Int Breastfeed J 2012, 7:12.

11. Friesen $H$, Vince J, Boas P, Danaya R, Mokela D, Ogle G, Asuo P, Kemiki A, Lagani W, Rongap T, Varughese M, Saweri W: Infant feeding practices in Papua New Guinea. Ann Trop Paediatr 1998, 18:209-215.

12. Benjamin A, Biddulph J: Port Moresby infant feeding survey. 1979. PNG Med J 1980, 23:92-96.

13. Frank D, Ripa P, Vince JD, Tefuarani N: Knowledge and attitudes about infant feeding among nulliparous and parous women in Port Moresby: a comparative study. PNG Med J 2008, 51(1-2):5-11.

14. 54th World Health Assembly: Infant and Young Child Nutrition (Resolution WHA54.2). Geneva: World Health Organization; 2001.

15. Ulak M, Chandyo RK, Mellander L, Shrestha PS, Strand TA: Infant feeding practices in Bhaktapur, Nepal: a cross-sectional, health facility based survey. Int Breastfeed J 2012, 7:1.

16. Lauer JA, Betrán AP, Victora CG, de Onís M, Barros AJD: Breastfeeding patterns and exposure to suboptimal breastfeeding among children in developing countries: review and analysis of nationally representative surveys. BMC Med 2004, 2:26.

17. Imdad A, Yakoob MY, Bhutta ZA: Effect of breastfeeding promotion interventions on breastfeeding rates, with special focus on developing countries. BMC Public Health 2011, 11(Suppl 3):S24.

18. World Health Organization: WHO HIV and Infant Feeding Technical Consultation Held on behalf of the Inter-agency Task Team (IATT) on Prevention of HIV Infections in Pregnant Women, Mothers and Their Infants. Consensus Statement. Geneva; 2006:25-27.

19. Duong DV, Binns CW, Lee AH: Breast feeding initiation and exclusive breast-feeding in rural Vietnam. Public Health Nutr 2004, 7(6):795-799.

20. Sudfeld CR, Fawzi WW, Lahariya C: Peer support and exclusive breastfeeding duration in low and middle-income countries: a systematic review and meta-analysis. PLoS One 2012, 7(9):e45143.

21. Reddy S: Breastfeeding - practices, problems \& prospects. J Fam Welfare 1995, 41(4):43-51.

22. Meyer-Rochow VB: Food taboos: their origins and purposes. J Ethnobiol Ethnomed 2009, 5:18.

23. Government of Papua New Guinea: National Health Plan 2011-2020: Volume 1 Policies and Strategies. Waigani, Papua New Guinea: Government of Papua New Guinea; 2010.

\section{Submit your next manuscript to BioMed Central and take full advantage of:}

- Convenient online submission

- Thorough peer review

- No space constraints or color figure charges

- Immediate publication on acceptance

- Inclusion in PubMed, CAS, Scopus and Google Scholar

- Research which is freely available for redistribution

Submit your manuscript at www.biomedcentral.com/submit 